\title{
Real-Time Evaluation Method of Vehicle Conflict Risk on Urban Expressway Based on Smartphone GPS Data
}

\author{
Le-Ning Wang ${ }^{1,2}$ \\ ${ }^{1}$ School of Mechanical and Automotive Engineering, Qingdao University of Technology, Qingdao 266520, China \\ ${ }^{2}$ Zhejiang Intelligent Transportation Engineering Technology Research Center, Hangzhou, Zhejiang 310030, China \\ Correspondence should be addressed to Le-Ning Wang; leningwang@163.com
}

Received 2 September 2021; Revised 29 September 2021; Accepted 4 October 2021; Published 18 October 2021

Academic Editor: Xinqiang Chen

Copyright (c) 2021 Le-Ning Wang. This is an open access article distributed under the Creative Commons Attribution License, which permits unrestricted use, distribution, and reproduction in any medium, provided the original work is properly cited.

To effectively evaluate the traffic safety risk of urban expressways in real time and ensure their traffic safety and smoothness, a realtime evaluation method of vehicle conflict risk of an urban expressway based on smartphone GPS data was proposed. We screened and processed smartphone GPS data to obtain vehicle behavior data, including acceleration and angular acceleration, and road state data, including average vehicle speed. Urban expressways were divided into four categories, closed straight section, closed curve section, vehicle entry section, and vehicle exit section; the evaluation indexes of abnormal vehicle behavior were established. Based on the improved entropy weight method, the vehicle conflict risk entropy was established to distribute the weight of different types of abnormal behaviors of vehicles. The evaluation system of vehicle conflict risk entropy was applied to the vehicle behavior data. Urban Expressways with more abnormal vehicle behavior were obtained to evaluate the risk of vehicle conflict in real time. The results showed that the easily obtained smartphone GPS data may be effectively used to analyze the abnormal behavior of vehicles, identify vehicle conflict risk points hidden in urban expressways in real time to provide effective methods for batch and dynamic real-time evaluations of vehicle conflict risks on urban expressways, and improve the traffic safety service level of urban expressways.

\section{Introduction}

With the recent emergence of mega cities and urban agglomerations, countries have actively adopted the construction of urban expressways to alleviate urban road traffic congestion and improve urban road traffic service levels $[1,2]$. Urban expressways connect main roads and intercity expressways in a city and are characterized by long distances, high traffic volumes, and high speed. These expressways occupy less than $6 \%$ of road traffic facilities but are responsible for greater than $30 \%$ of standard vehicle kilometers [3]. However, to meet the travel needs of residents as much as possible in the planning and design of urban expressways, the road characteristics of large numbers of imports and exports, small spacing, and complex road networks, which further lead to rapid changes in vehicle speed and a high frequency of vehicle lane changes that result in vehicle conflict risk and road traffic accidents, must be considered. According to a statistical report on road traffic accidents in China in 2017, there were an average of 554 road traffic accidents on urban expressways every month, which resulted in nearly 140 deaths and 572 injuries [4]. Therefore, real-time vehicle conflict risk assessments on urban expressways and real-time corresponding traffic control on sections with excessive vehicle conflict risk would effectively improve the traffic service level of urban expressways.

Current research on vehicle conflict risk has achieved fruitful results in assessing road traffic safety and may be primarily divided into the following two aspects:

(1) Data-driven: Based on the large number of vehicle collision data and road traffic accident data collected, based on improved neural network or Logit model, or via the computer processing of video images, the risk prediction of vehicle conflict may be realized.

(2) Model-driven: The related factors that may affect the risk of vehicle conflict, such as vehicle movement, 
road environment, and residents' travel conditions, were analyzed. Support vector machine (SVM) and Bayesian methods were used to establish 3D collision models or multivariate comprehensive evaluation models to evaluate vehicle conflict risk.

Studies of vehicle conflict risk using data-driven assessments. Liu and Chen [5] proposed a fuzzy reasoning model based on a decision tree and adaptive neural network to predict the risk of vehicle conflict in urban expressways in real time. Sun et al. [6] considered the collision mechanism under different road congestion conditions and the correlation between vehicle collision and space; they established three types of collision models and two spatial models, respectively, and adopted a Bayesian method to determine relevant parameters. Strickland et al. [7] performed in-depth learning on traffic accident video streams via the Bayesian convolution long short-term memory (LSTM) method to realize the prediction of impending accidents. Basso et al. [8] realized expressway accident prediction based on a random forest program, support vector machine, and logistic regression using the vehicle data collected with an automatic vehicle identification function at a free flow toll station. Hossain and Muromachi [9] evaluated the traffic safety of ramp vehicles on urban expressways based on a random polynomial logit model, and the prediction of vehicle collision was realized based on the Bayesian belief network model. Hassan and Abdel-Aty [10] studied the relevant factors of vehicle collision under reduced visibility. The research showed that nearly $70 \%$ of vehicle collisions related to visibility may be correctly identified. Hong et al. [11] applied a double hurdle approach to analyze the risk factors of multivehicle collision. Fountas et al. [12] developed a random parameter logit framework to study the comprehensive impact of dynamic and static factors on traffic conflict and considered the correlation between random parameters. Kassu and Hasan [13] considered the impact of the number of lanes on vehicle collisions on urban roads and applied the negative binomial regression method to obtain the key factors causing fatal and nonfatal injuries to road traffic.

There are some studies on vehicle conflict risk using model-driven assessments. Wang et al. [14] used Bayesian logistic regression and a support vector machine model to explore the impact of the number of residents and travel conditions on the vehicle collision risk of expressway ramps. $\mathrm{Wu}$ et al. [15] used a simulated vehicle network physical system (VCPS), based on the Kalman filter (KF) algorithm, combined with driver behavior and road geometry information to detect vehicle collision risk. Habtemichael and De Picado Santos [16] used a microtraffic simulation method and VISSIM simulation software to quantitatively evaluate the safety impact of aggressive driving behavior. Katrakazas et al. [17] developed a joint framework of interactive perception motion model and dynamic Bayesian network to perceive vehicle collision risk. Hosseinpour et al. [18] used multivariate Poisson log-normal model with spatial correlation to predict multivehicle collision frequency. The time factor had a great impact on collision, and Chen et al. [19] developed the unbalanced panel data hybrid logit model to study the possibility of hourly collision on expressways and confirmed that real-time weather conditions, road conditions, and traffic conditions had a significant impact on the occurrence of vehicle conflict.

However, some imperfections in previous research methods remain. (1) The evaluation and prediction of vehicle collision based on historical accident data do not take into account the differences in vehicle movement behaviors at different dates and time periods. Therefore, it cannot accurately evaluate the risk of vehicle collision and road safety based on the current actual situation, which results in poor timeliness. (2) The real-time data acquired by road-side units and lane cameras are small and have poor continuity, which makes it impossible to accurately judge the specific speed and lane changes of each vehicle and has a low recognition rate. The comprehensive evaluation method combined with various factors could not achieve real-time and accurate evaluations, and it tended to overconsider the impact of environment on vehicle conflict without considering the specific motion state of vehicles, which resulted in a low accuracy rate.

With the continuous development of intelligent transportation systems and mobile devices [20], some scholars used vehicle OBD or GPS data for vehicle conflict assessments. Stipancic et al. [21] used vehicle GPS travel data from Quebec City to extract vehicle hard braking and acceleration information and then compared it with historical crash data for evaluation. The results showed that the number of vehicle acceleration and braking incidents was directly related to the severity of the collision. However, the above literature only analyzed changes in vehicle speed without judging or evaluating the actual conditions of specific roads. Therefore, it was not applicable to actual urban expressways or some specific road conditions. Cai et al. [22] established road traffic safety entropy based on on-board OBD data to determine the correlation between abnormal driving behaviors and road traffic accident points. However, the on-board OBD did not have Internet communication, and it could not make real-time and accurate statistics of the movement state data of vehicles on the road to evaluate the risk of vehicle conflict in real time.

Therefore, the present article used driver smartphone real-time GPS data to obtain the vehicle behavior information. We analyzed the impact of the dangerous movement state of vehicles in different sections of urban expressways on vehicle conflict and divided the urban expressway to establish a vehicle conflict risk entropy evaluation model and realize the real-time evaluation of vehicle conflict risk on urban expressways.

\section{Research Method}

(1) Collection of vehicle satellite positioning data in real time using the GPS module of drivers' smartphones. The data were bound to the road to accurately correspond to the actual road coordinates to sort, 
filter, and calculate the data and obtain vehicle information and traffic information, such as vehicle speed, acceleration, angular velocity, angular acceleration, and average vehicle speed.

(2) Analyses of the traffic and vehicle behavior characteristics of urban expressway and evaluation of the possible collision types of vehicles. The urban expressway was divided into a closed straight section, closed curve section, vehicle entry section, and vehicle exit section. According to the different effects of abnormal vehicle behaviors on collision types in different sections, the evaluation indexes of abnormal vehicle behaviors, including rapid acceleration and deceleration, were established.

(3) Construction of traffic conflict risk entropy using the improved entropy method with vehicle behavior as indicators, to establish traffic conflict risk entropy in different types of urban expressway road vehicles of all kinds of abnormal behavior to determine the vehicles with various kinds of abnormal behavior for a specific road vehicle's risk degree of the impact of the conflict.

(4) Using the established vehicle conflict risk entropy and based on the GPS data of online ride-hailing in Chengdu city, the vehicle conflict risk levels of different types of sections of urban expressways were evaluated in real time. We established the thermal map of vehicle conflict risk level of urban expressways and ultimately determined the vehicle conflict risk concentration point of the Second Ring Expressway in Chengdu city to provide vehicle conflict risk early warnings for drivers and reasonable suggestions for traffic management departments.

\section{Data Acquisition and Processing}

3.1. Data Acquisition and Preprocessing. The results and discussion may be presented separately, or in one combined section, and may optionally be divided into headed subsections.

3.2. Advice on Equations. With the continuous development of "Internet + transportation," urban public transportation services have gradually shifted from the traditional "taxi" to a diversified mode of "online ride-hailing + taxi." According to statistics from China's online ride-hailing regulatory information exchange platform, Chengdu city ranks first and second among China's 36 central cities in the number of licenses and orders for online ride-hailing vehicles, respectively. A large amount of vehicle satellite positioning data is generated daily in Chengdu city, Sichuan Province, China based on the GPS module in the smartphones of online ride-hailing drivers.

As shown in Figure 1, each green dot represents the vehicle GPS data of the online ride-hailing platform in northeast Chengdu city on October 15, 2016 (Coordinate range [30.727818, 104.043333], [30.726490, 104.129076] [30.655191, 104.129591], [30.652828, 104.042102]). Vehicle

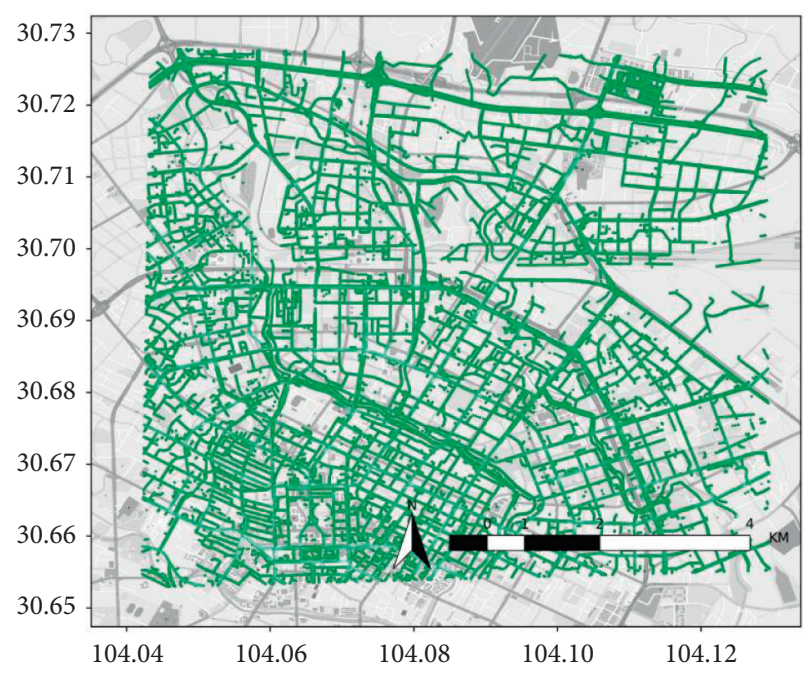

FIgURE 1: GPS data of online ride-hailing in Chengdu city.

GPS data of the network ride-hailing platform are obtained. The original data set contained driver information, order number information, UNIX timestamp information, and vehicle longitude and latitude coordinates, which were collected every $2-4 \mathrm{~s}$. There were approximately 1.8 billion pieces of monthly statistical data, with a total size of approximately $88.04 \mathrm{~GB}$.

To comply with legal provisions and use relevant data reasonably and effectively, the following preliminary processing was performed on the collected data. (1) The GPS coordinate information was bound to the road. Positioning in the civil GPS module often has a certain coordinate offset, which does not ideally correspond to the actual road position. To ensure that the GPS data of the driver's smartphone accurately corresponded to the actual roads in Chengdu city, the collected longitude and latitude coordinates were bound to the road. (2) Personal information was processed. From the vehicle GPS data, the departure and destination coordinates of each online ride-hailing order were reflected. Therefore, to protect the personal information of drivers and passengers, driver identification (ID) and order ID were encrypted. To ensure the privacy of passengers, the coordinates of passenger origin point and destination point were removed. Some online ride-hailing order information and data extracted after preprocessing are shown in Table 1.

3.3. Data Processing. Cleaning and screening for the collected data. For data missing from some nodes generated due to smartphone network conditions and other reasons, the weighted average algorithm is used to complete the missing data to achieve data continuity. In order to avoid the influence of such data on the average speed of the vehicle, the data of temporary vehicle parking caused by boarding and boarding passengers, traffic accidents, and drivers' or passengers' subjective intentions are eliminated.

Processing of the cleaned and screened data. The cleaned and filtered data were converted using the following 
TABle 1: Sample of ride-hailing data.

\begin{tabular}{|c|c|c|c|c|}
\hline Driver ID & Order ID & Unix & Longitude $\mathrm{Lo}_{i j}$ & Driver ID $\mathrm{La}_{i j}$ \\
\hline $\mathrm{eb} 4, \ldots, 2 \mathrm{bf}$ & $660, \ldots, 959$ & 1476462916.00 & 104.0681 & 30.65824 \\
\hline$e 5 c, \ldots, 9 f 8$ & $\mathrm{c} 29, \ldots, \mathrm{c} 38$ & 1476510400.00 & 104.0659 & 30.65853 \\
\hline $\mathrm{e} 7 \mathrm{~d}, \ldots, \mathrm{a} 3 \mathrm{~b}$ & $150, \ldots, 70 f$ & 1476537902.00 & 104.0710 & 30.65721 \\
\hline $213, \ldots$, ad 7 & $862, \ldots, 8 c 6$ & 1476520440.00 & 104.0791 & 30.66735 \\
\hline $000, \ldots, \mathrm{e} 06$ & $428, \ldots, 62 \mathrm{e}$ & 1476491503.00 & 104.0453 & 30.65924 \\
\hline $000, \ldots, 34 b$ & $80 b, \ldots, f 50$ & 1476503116.00 & 104.1104 & 30.67071 \\
\hline
\end{tabular}

methods to reflect the vehicle motion state, including speed, acceleration, angular speed, and angular acceleration, and the road traffic state, such as the average speed of vehicles on the road.
Equation (1) was used to obtain the vehicle displacement between two time nodes using the vehicle longitude and latitude coordinates at time $n$ and time $n+1$.

$$
\begin{aligned}
& \qquad S_{i j}^{n}=\arccos \left[\cos \left(90-\mathrm{La}_{i j}^{n+1}\right) \cdot \cos \left(90-\mathrm{La}_{i j}^{n}\right)+\sin \left(90-\mathrm{La}_{i j}^{n+1}\right) \cdot \sin \left(90-\mathrm{La}_{i j}^{n}\right) \cdot \cos \left(\mathrm{Lo}_{i j}^{n+1}-\mathrm{Lo}_{i j}^{n}\right)\right], \\
& \text { where } \mathrm{Lo}_{i j}^{n} \text { and } \mathrm{La}_{i j}^{n} \text { represent the longitude and latitude } \quad \theta_{i j}^{n}=\arccos \left(-\frac{\left(X_{i j}^{n}\right)^{2}+\left(X_{i j}^{n+1}\right)^{2}-\mathrm{Dist}^{2}(n-1, n+1)}{2 X_{i j}^{n} X_{i j}^{n+1}}\right), \\
& \text { coordinates of the vehicle, respectively, when the driver in }
\end{aligned}
$$
the $i$ th place executes Order No. $J$ at the time $n$.

As shown in Figure 2, because of the time node difference of the collected data is $3 \mathrm{~s}$ compared with the complete movement time of the vehicle, the vehicle displacement may be approximately equal to the forward distance of the vehicle in a short period.

Equation (2) was used to obtain the displacement of vehicle movement at each time interval as approximated $X_{i j}^{n}$.

$$
X_{i j}^{n} \approx S_{i j}^{n}
$$

Equations (3) and (4) were used to obtain the instantaneous speed and acceleration of the vehicle, which were further determined.

$$
\begin{gathered}
V_{i j}^{n}=\frac{X_{i j}^{n}}{\Delta T^{n}}, \\
a_{i j}^{n}=\frac{V_{i j}^{n}}{\Delta T^{n}} .
\end{gathered}
$$

Vehicle lateral movement is one of the main causes of vehicle conflict [23-25]. To effectively evaluate the relationship between vehicle behavior and vehicle conflict, it was necessary to further determine vehicle angular velocity and angular acceleration. To obtain the change in vehicle instantaneous azimuth $\theta_{i j}^{n}$, from equation (1), the vehicle displacement between two time nodes were obtained using the vehicle longitude and latitude coordinates at time $n$ and time $n+1$, as shown in Figure 3 .

Substituting the displacement $X_{i j}^{n}$ and $X_{i j}^{n+1}$ in the $n$ and $n+1$ segments calculated from equation (1) into equation (5), the instantaneous change in azimuth angle of the vehicle $\theta_{i j}^{n}$ was calculated. where $\operatorname{Dist}^{2}(n-1, n+1)$ represents the displacement between the $n-1$ time node and $n+1$ time node.

According to equations (6) and (7), the instantaneous angular velocity and angular acceleration of the vehicle were further determined.

$$
\begin{aligned}
& \omega_{i j}^{n}=\frac{\theta_{i j}^{n}}{\Delta T}, \\
& \partial_{i j}^{n}=\frac{\omega_{i j}^{n}}{\Delta T^{n}} .
\end{aligned}
$$

In addition to the vehicle's own behavior, the road traffic conditions, including the average vehicle speed, will also affect the frequency and probability of vehicle conflict. The online ride-hailing data collected in this article generate about 60 million vehicle positioning data every day. Because the average time interval of data collection is $3 \mathrm{~s}$, it represents that in the northwest of Chengdu city, the instantaneous online ride-hailing operation number is nearly 2800 based on the daily online ride-hailing operation of $12 \mathrm{~h}$. Therefore, the short-term average speed of online ride-hailing can effectively represent the average speed of vehicles in each section of the Second Ring Expressway in Chengdu city. According to equation (8), the average vehicle speed $\overline{V_{i j}^{n}}$ of this section was obtained at the $n$ time node, as shown in Figure 4 .

$$
\overline{V_{i j}^{n}}=\frac{\sum_{i j} V_{i j}^{n}}{i+j},
$$

where $\sum_{i j} V_{i j}^{n}$ represents the sum of the average speeds of all vehicles in the microsegment. 


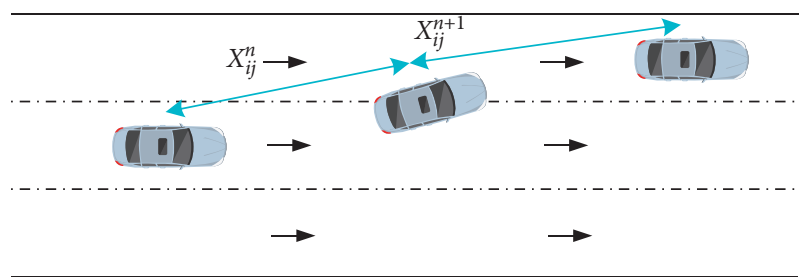

Figure 2: Vehicle instantaneous displacement.

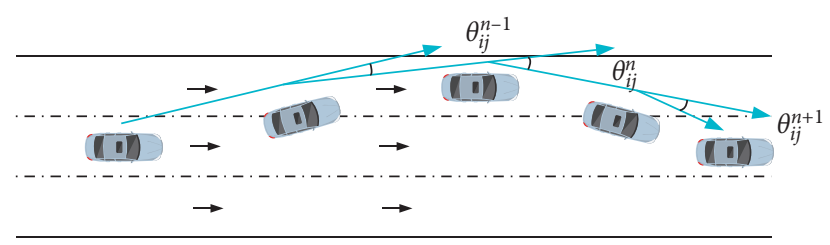

Figure 3: Vehicle instantaneous angular displacement.

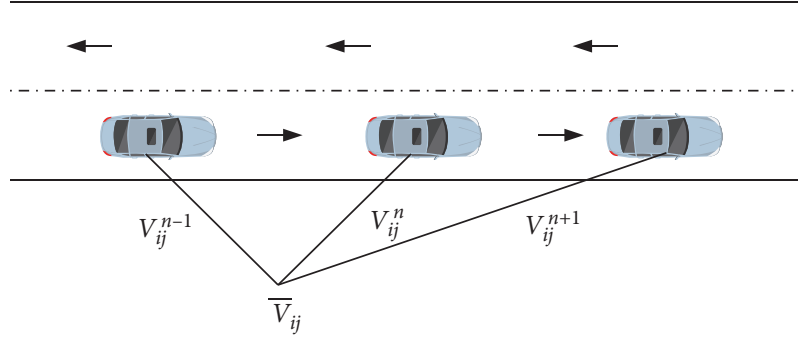

Figure 4: Average vehicle speed.

Based on NumPy, Pandas, and other functions in the Python programming language, the above methods were used to process online ride-hailing order information and original data to obtain vehicle motion behavior data. The data processing example is shown in Table 2.

\section{Establishment of the Division of Vehicle Conflict Risk Sections on Urban Expressways and Vehicle Conflict Risk Evaluation System}

There were numerous correlations between different types of road vehicle conflict risk and vehicle behavior, including the characteristics of the road section itself, such as speed limit and road shape, which affected vehicle behavior to a certain extent. As shown in Figure 5, based on the calculated vehicle behavior data, by classifying and defining the road section characteristics and possible vehicle behavior, the urban expressway was divided into four categories: closed straight section, closed curve section, vehicle entry section, and vehicle exit section.

\subsection{Classification of Urban Expressway Section Types}

(1) Closed straight sections (as shown in I in Figure 5) are most widely used in expressway design due to high vehicle traffic efficiency and low construction cost. However, according to the statistics of all kinds of linear traffic accidents in China, the traffic accident rate of straight road sections was high. An in-depth analysis of vehicle behavior revealed primarily two reasons: (1) Vision is wide, the road is flat, and the road traffic condition is good in the straightline section. The driver will inadvertently increase the vehicle speed, relax his vigilance, and reduce their observation of adjacent lanes and nearby vehicles. (2) The discrete speed of vehicles in straight-line sections is large, which results in frequent overtaking [26].

As shown in Figure 6, the analyses of the instantaneous displacement, velocity, acceleration, change of instantaneous azimuth, angular velocity, and angular acceleration of vehicles in the selected closed straight section showed that for the closed straight section of the urban expressway, the average speed of vehicles was mostly less than $15 \mathrm{~m} / \mathrm{s}$, and the range of vehicle longitudinal acceleration was widely distributed with a high degree of dispersion. For the lateral changes of vehicle, the angular velocity and angular acceleration were below $5 \mathrm{rad} / \mathrm{s}$, and the amount of left-hand angle change was roughly equal to right-hand angle change, which was consistent with the actual situation of vehicle lane change and overtaking.

(2) Closed curve section (as shown in II in Figure 5), as a section type of urban expressway, was primarily used near the four vertices of the circular expressway. Statistics showed that the severity of traffic accidents caused by curved sections was higher than straight sections. The following main causes of curve traffic accidents were identified: (1) when the vehicle drives into the curve from the closed straight road section, the field of vision gradually narrows, and the driver engages in stress behaviors, such as deceleration, after the field of vision narrows, which impacts other vehicles. (2) After the vehicle enters the curve, the driver needs to constantly adjust the steering wheel to keep the vehicle driving along the track of the lane boundary. If the steering wheel is not adjusted in time or over adjusted, the vehicle will deviate from the normal driving route.

As shown in Figure 7, the positive acceleration of vehicles in curve sections was lower than that in the closed straight section, but the negative acceleration was higher than that in the closed straight section. Therefore, the impact of vehicle deceleration on vehicle conflict was greater than that in closed straight section. For the lateral movement of vehicles, the direction of angular change and the lateral movement direction of the vehicles were the same because the intercepted section was a one-way turning section.

(3) Vehicle entry section (as shown in III in Figure 5): Vehicles enter the main road via ramps and acceleration lanes. The following main reasons for potential traffic safety hazards were identified. (1) For vehicles entering the main road, oppressive or cooperative behavior was used to merge into the main 
TABLe 2: Sample vehicle behavior data.

\begin{tabular}{lcccccc}
\hline Drivers ID & Order ID & $X_{i j}^{n}$ & $V_{i j}^{n}$ & $a_{i j}^{n}$ & $\theta_{i j}^{n}$ & $\omega_{i j}^{n}$ \\
\hline $000, \ldots, \mathrm{e} 06$ & $072, \ldots, 887$ & 19.93 & 6.643 & -1.23 & 3.14 & 0.02 \\
$000, \ldots, \mathrm{e} 06$ & $072, \ldots, 887$ & 31.57 & 10.523 & 1.293 & 3.1 & -0.01 \\
$000, \ldots, \mathrm{e} 06$ & $072, \ldots, 887$ & 38.21 & 12.737 & 0.738 & 0 & -1.03 \\
$000, \ldots, \mathrm{e} 06$ & $072, \ldots, 887$ & 30.17 & 10.057 & -0.893 & 3.14 & 1.05 \\
$000, \ldots, 34 \mathrm{~b}$ & $698, \ldots, 190$ & 21.99 & 7.33 & -0.29 & 2.61 & 0.01 \\
$000, \ldots, 34 \mathrm{~b}$ & $698, \ldots, 190$ & 24.28 & 6.07 & -0.315 & 3.05 & 0.34 \\
$000, \ldots, 34 \mathrm{~b}$ & $698, \ldots, 190$ & 15.41 & 5.137 & -0.311 & 3.1 & -0.11 \\
\hline
\end{tabular}

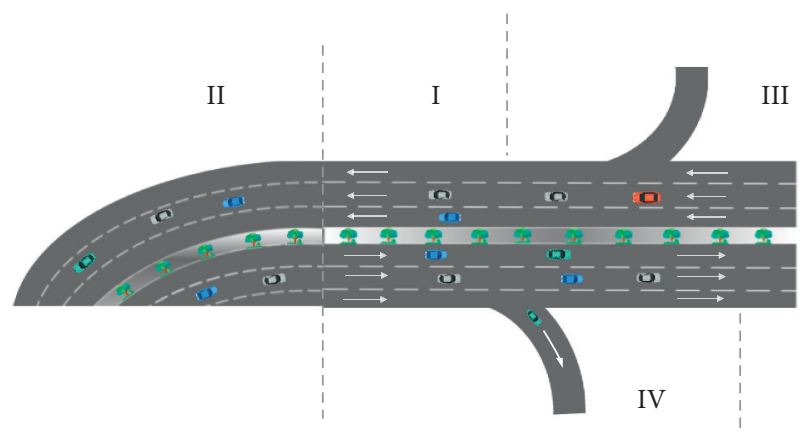

Figure 5: Urban expressway section type division.

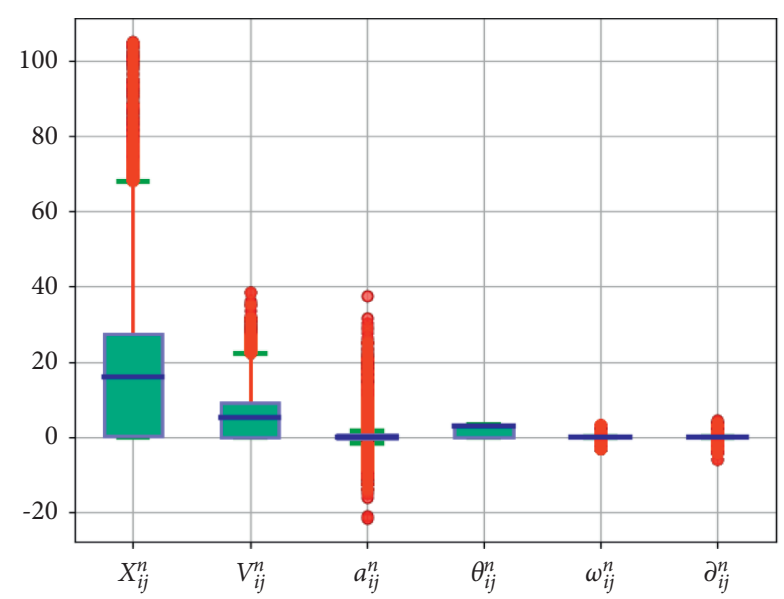

Figure 6: Box diagram of vehicle behavior in the closed straight section.

road, which affected the normal vehicles in the main road. (2) The normal running vehicles on the main road slow down and avoid or accelerate due to the entry of vehicles on the right side, which have a certain impact on the normal running of vehicles behind.

As shown in Figure 8, the lateral collision frequency of vehicles increased in this section, and the change in negative angular velocity and angular acceleration was greater than the positive angular velocity and angular acceleration due to the entry of vehicles.

(4) Vehicle exit section (as shown in IV in Figure 5): The vehicle enters the deceleration lane via lane change or continuous lane change and drives out of the urban expressway. The following main reasons for the existence of potential traffic safety hazards were identified. (1) For outgoing vehicles, lane change and continuous lane change caused potential safety hazards to other normal vehicles on the main road. (2) For vehicles that did not plan to leave via this exit, they were oppressed by the right direction due to the lane change of vehicles, which resulted in the forced deceleration or lane change of vehicles and caused safety risks to themselves and other vehicles.

As shown in Figure 9, the lateral collision frequency of vehicles increased in this section, and the change in positive angular velocity and angular acceleration were greater than the negative angular velocity and angular acceleration due to the departure of vehicles.

\section{Establishment of Vehicle Behavior Evaluation System for Urban Expressway}

The in-depth analysis of the types of urban expressway sections and the hidden dangers of road traffic safety was used to establish the vehicle behavior evaluation system of urban expressway using the closed straight sections, closed curve sections, vehicle entry sections, and vehicle exit sections as the primary indicators and vehicle behavior, such as vehicle acceleration, and angular acceleration, as the secondary indicators, as shown in Figure 10.

The vehicle will have large longitudinal and transverse speed changes in the closed straight section due to overspeed, lane change, and other behaviors. Therefore, vehicle acceleration (E11), deceleration (E12), left and right angular acceleration (E13), and vehicle speed greater than the average vehicle speed (E14) were secondary indicators of vehicle behavior in the closed straight road section (E1).

Vehicles reduce their longitudinal speed and change transverse speed in the closed curve section due to deceleration, steering, and other behaviors. Therefore, vehicle deceleration (E21), vehicle left and right angular acceleration (E22), and vehicle speed greater than the vehicle average speed rate (E23) were the secondary indicators of vehicle behavior in the closed curve section (E2).

Vehicles reduce their longitudinal speed and change lateral speed to the left in the vehicle entry section due to deceleration, avoidance, entering vehicles, and lane changing. Therefore, vehicle deceleration (E31), vehicle left angular acceleration (E32), and speed greater than the vehicle average speed rate (E33) were the secondary indicators of vehicle behavior in the vehicle entry section (E3). 


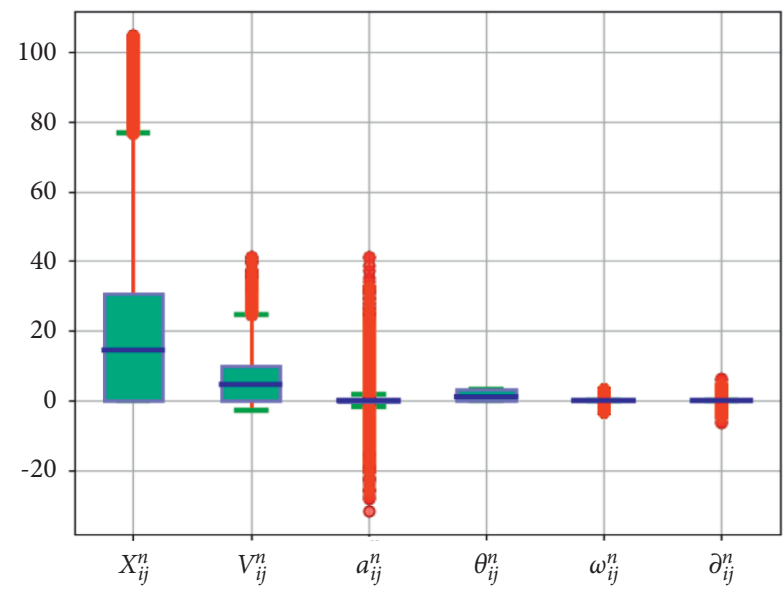

Figure 7: Box diagram of vehicle behavior in the closed curve section.

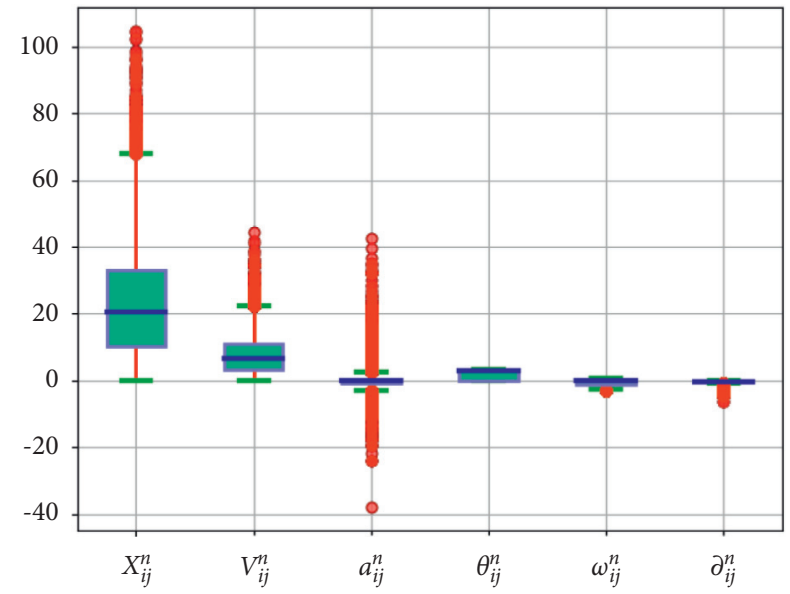

Figure 8: Box diagram of vehicle behavior in the vehicle entry section.

The longitudinal speed reduction and lateral speed change in the right direction occur in the vehicle exit section due to the deceleration of the vehicle and the lane change of some outgoing vehicles. Therefore, vehicle deceleration (E41), vehicle right angular acceleration (E42), and vehicle speed greater than the vehicle average speed rate (E43) were the secondary indicators of the vehicle behavior in the section where the vehicle drives out (E4).

\section{Vehicle Conflict Risk Entropy Model}

6.1. Model Building. As a measure of system disorder, entropy reflects the amount of useful information carried by each index. The greater the amount of information carried by each index, the smaller the entropy, and the smaller the amount of information carried, the greater the entropy. The entropy weight method assigns weight to each index according to the impact of the relative transformation degree of each evaluation index on the evaluation system. This model has been widely used in various comprehensive evaluations $[27,28]$. The evaluation system of vehicle conflict behavior established in this article mainly includes the comprehensive evaluation of vehicle longitudinal acceleration, angular acceleration, and other behaviors. Therefore, the applicability and rationality of the vehicle conflict risk entropy evaluation model are mainly due to the following two aspects: (1) Entropy weight method judges the discretization degree of an index through entropy value. It is considered that the higher the discretization degree of an index, the greater its role in the whole evaluation system. In the process of vehicle movement, the greater the change of vehicle longitudinal acceleration or transverse acceleration, the higher the probability of vehicle conflict risk. (2) During the application of entropy weight method because it judges the dispersion degree of an index through entropy, the amount of entropy data will have a great impact on the evaluation results. When the amount of data are insufficient, it will have a certain impact on the judgment results. The amount of vehicle behavior data used in this study is huge, which can effectively and accurately evaluate the risk of vehicle conflict. Therefore, the establishment of an improved entropy weight evaluation model can comprehensively evaluate the impact of vehicle behavior on vehicle conflict.

The traditional entropy weight method is a measure of the degree of deviation between the index and the ideal solution. However, among the indicators of vehicle conflict risk, the degree of vehicle velocity dispersion, vehicle acceleration dispersion, and vehicle overspeed dispersion have different effects on the risk of vehicle conflict and are related to some extent. Therefore, this model improves the entropy weight method by taking $85 \%$ of the maximum value of vehicle speed and angular velocity as the evaluation standard to adjust the two evaluation indexes, as shown in equations (9) and (10):

$$
\begin{gathered}
\delta P_{v}=\frac{\left(v_{i j}\right)_{n \times m}}{\left(\left(v_{i j}\right)_{n \times m} \mathrm{MAX}\right) * 0.85}, \\
\delta P_{\omega}=\frac{\left(\omega_{i j}\right)_{n \times m}}{\left(\left(\omega_{i j}\right)_{n \times m} \mathrm{MAX}\right) * 0.85} .
\end{gathered}
$$

According to equation (11), the corresponding evaluation matrix $\mathrm{P}$ is constructed based on the constructed evaluation index of vehicle conflict behavior on urban expressways.

$$
P=\left(p_{i j}\right)_{n \times m},
$$

where $p_{i j}$ is the $j$ evaluation index of sample No. I.

To further eliminate the influence of different index dimensions, the evaluation indexes were standardized. For the vehicle conflict evaluation indexes, including vehicle longitudinal acceleration and angular acceleration, the vehicle is considered best in the stationary state, and equation (12) was used to consider the indexes greater than zero, such as longitudinal acceleration and angular acceleration. Equation (13) was used to process the indexes less than zero, such as longitudinal deceleration, to obtain the standardized evaluation matrix $B_{i j}$. 


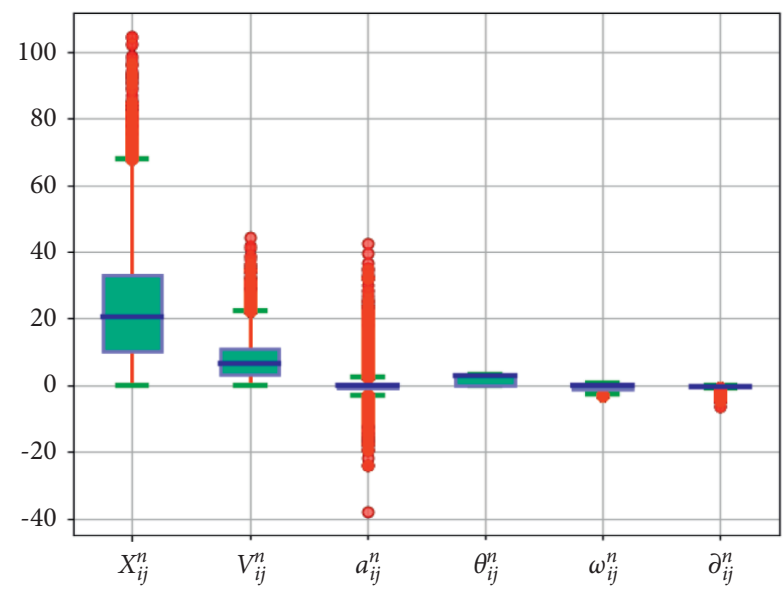

FIGURE 9: Box diagram of vehicle behavior in the vehicle exit section.

$$
\begin{gathered}
b_{i j}=\frac{\max _{j}\left(p_{i j}\right)-p_{i j}}{\max _{j}\left(p_{i j}\right)-\min _{j}\left(p_{i j}\right)}, \\
b_{i j}=\frac{p_{i j}-\min _{j}\left(p_{i j}\right)}{\max _{j}\left(p_{i j}\right)-\min _{j}\left(p_{i j}\right)} .
\end{gathered}
$$

Equation (14) was used to calculate the characteristic proportion $Z_{i j}$ of the $i$ th evaluation object under the $j$ th evaluation index.

$$
Z_{i j}=\frac{b_{i j}}{\sum_{i=1}^{n} b_{i j}} .
$$

In equation (15), the entropy value $e_{j}$ of the $j$ th index may be obtained as follows:

$$
e_{j}=-\frac{1}{\ln n} \sum_{i=1}^{n} Z_{i j} \ln Z_{i j} \text {. }
$$

Equation (16) was used to obtain the coefficient $g_{j}$ of differentiation of the $j$ th index.

$$
g_{j}=1-e_{j}
$$

Using equation (17), the weight coefficient of the evaluation index $w_{j}$ of item $j$ was finally obtained:

$$
w_{j}=\frac{g_{i}}{\sum_{i=1}^{m} g_{i}} .
$$

6.2. Model Application. The entropy evaluation model of abnormal vehicle behavior was applied to the processed vehicle behavior data. Because the data volume of all of the data was too large, it was difficult to directly use it to calculate the weight coefficient of the evaluation index. Therefore, the random sampling method was used. According to the divided road section types, three groups of 100,000 lines of data were selected for the four types of road sections, and the entropy weight method was used to evaluate these data. Equation (18) was used to analyze the accuracy of the evaluation results of each group according to the root mean squared error. If the error was within a reasonable range, the result was considered effective.

$$
\text { RMSE }=\sqrt{\frac{1}{m} \sum_{i=1}^{m}\left(\bar{y}-y_{i}\right)^{2} .}
$$

(1) The comprehensive evaluation results of the closed straight section are shown in Table 3. Figure 11 shows that the longitudinal acceleration of vehicles had the greatest impact on road traffic safety in closed straight sections and accounted for $46.22 \%$. The longitudinal deceleration of vehicles had the second impact on road traffic safety and accounted for $23.07 \%$. The left and right angular acceleration and speed greater than the average speed of vehicles accounted for $18.44 \%$ and $12.25 \%$, respectively.

(2) The comprehensive evaluation results of closed curve sections are shown in Table 4 . Figure 12 shows that the longitudinal deceleration of vehicles had the greatest impact on road traffic safety in the closed curve section and accounted for $98.42 \%$. The rates of the left and right angular acceleration and speed of vehicles were greater than the average speed of vehicles and accounted for only $0.67 \%$ and $0.89 \%$, respectively, which had little impact on road traffic safety.

(3) The comprehensive evaluation results of the vehicle entry section are shown in Table 5 . Figure 13 shows that the left angular acceleration of vehicles had the greatest impact on road traffic safety in the section where vehicles converged and accounted for $70.78 \%$. The rates of vehicle longitudinal deceleration and vehicle speed greater than the average speed of vehicles accounted for $18.35 \%$ and $11.15 \%$, respectively.

(4) The comprehensive evaluation results of the vehicle leave section are shown in Table 6. Figure 14 shows that the right-hand angular acceleration of the vehicle had the greatest impact on road traffic safety in the section where vehicles leaved and accounted for $69.77 \%$, and the rates of vehicle longitudinal deceleration and vehicle speed greater than the average vehicle speed accounted for $18.95 \%$ and $11.27 \%$, respectively.

\section{Expressway Real-Time Traffic Conflict Risk Points}

Using equation (19), based on the entropy evaluation model of the abnormal behavior of vehicles on urban expressways, the real-time evaluation of vehicle conflict risk level was performed for different sections of the urban expressway.

$$
\mathrm{Val}=\sum \sum_{j=1}^{m} w_{j} b_{i j} .
$$




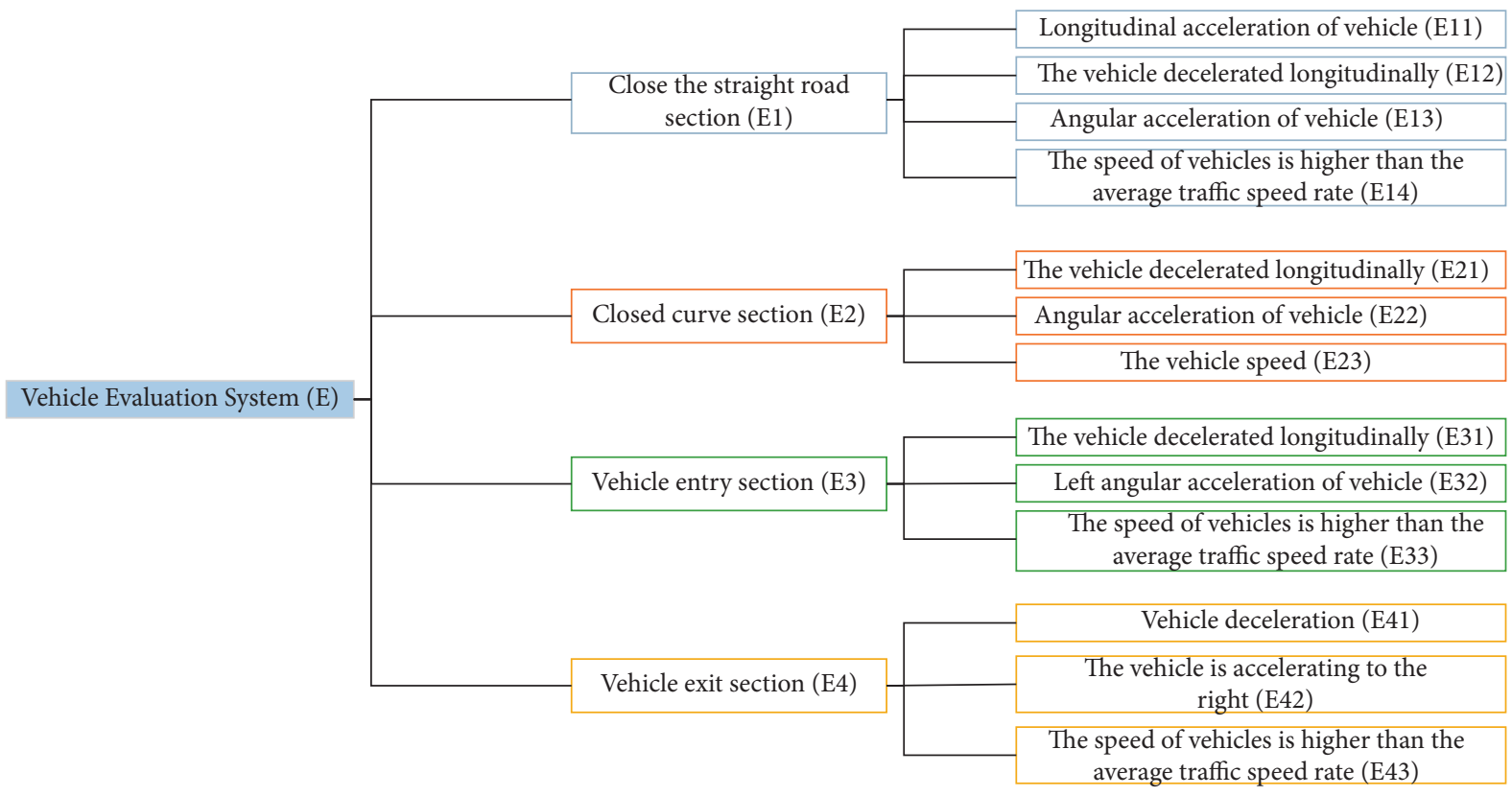

Figure 10: Vehicle behavior evaluation system.

TABLE 3: Evaluation result of closed straight section.

\begin{tabular}{lcccc}
\hline & $(\mathrm{E} 11)$ & $(\mathrm{E} 12)$ & $(\mathrm{E} 13)$ & $(\mathrm{E} 14)$ \\
\hline Test1 & 0.350447 & 0.250286 & 0.238371 & 0.160897 \\
Test2 & 0.544678 & 0.216923 & 0.119302 & 0.119098 \\
Test3 & 0.491546 & 0.225020 & 0.195811 & 0.087623 \\
Result & 0.462224 & 0.230743 & 0.184495 & 0.122539 \\
RMSE & 0.081963 & 0.014213 & 0.049264 & 0.030016 \\
\hline
\end{tabular}

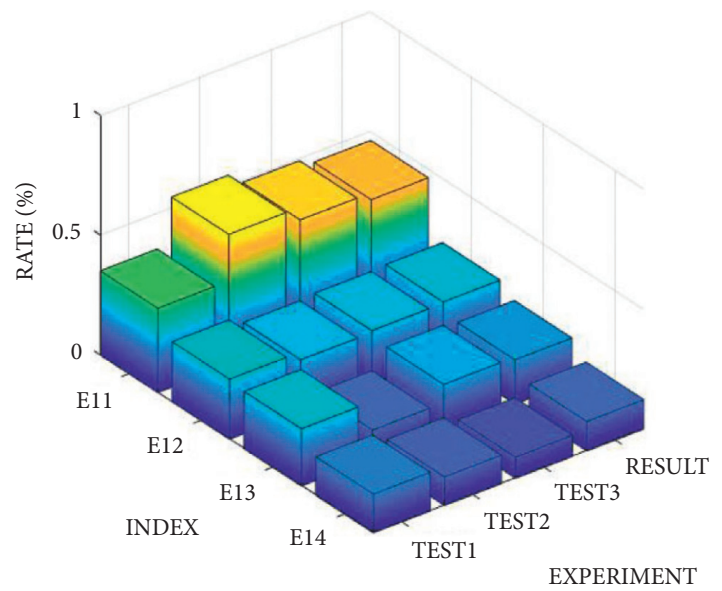

Figure 11: Proportion of each index of closed straight section.

Table 4: Proportion of each index of closed curve section.

\begin{tabular}{lccc}
\hline & $(\mathrm{E} 21)$ & $(\mathrm{E} 22)$ & $(\mathrm{E} 23)$ \\
\hline Test1 & 0.981896 & 0.007565 & 0.010540 \\
Test2 & 0.981334 & 0.008788 & 0.009878 \\
Test3 & 0.989590 & 0.003964 & 0.006446 \\
Result & 0.984273 & 0.006772 & 0.008955 \\
RMSE & 0.003766 & 0.002049 & 0.001794 \\
\hline
\end{tabular}

In the formula, the obtained evaluation index weight $w_{j}$ was used to sum the various behaviors $b_{i j}$ of each vehicle, and the sum of abnormal vehicle behaviors in each small section was ultimately determined to calculate the real-time evaluation of vehicle conflict risk in various fast sections.

7.1. Change Trend of Daily Conflict Risk. In order to effectively and intuitively analyze the above experimental results, the vehicle conflict risk heat maps of various road sections on October 15, 2016 are selected, in which the ordinates of Figures 15-18 represent UNIX timestamp information and the abscissa represents the longitude coordinates of the road section. By analyzing heat maps of vehicle conflict risk in four sections, the daily variation trend of vehicle conflict risk in different sections of urban expressways can be obtained.

Figure 15 shows that the risk of vehicle conflict in closed straight sections began to increase around 8 a.m., reached the peak of the day at 11 a.m., then decreased slowly and remained stable to about 4 p.m. With the beginning of the evening peak, the risk of vehicle conflict reached another peak at 7 p.m., then remained stable and decreased slowly.

Figure 16 shows that the risk of vehicle conflict in closed curve sections began to increase at 8 a.m., increased rapidly at 10 a.m., reached the peak of the day at 2 p.m., remained stable to about 7 p.m., and then decreased slowly.

According to Figures 17 and 18, the traffic flow of vehicles entering and leaving the road was less than the main road. However, the time node of vehicle conflict risk was similar to the closed straight sections. The risk started to increase at about 7 a.m., reached the peak of the day at 10 a.m., then slowly decreased and remained stable to about 4 p.m. With the beginning of the evening peak, the vehicle conflict risk reached another peak at 6 p.m. and then remained stable and slowly decreased. 


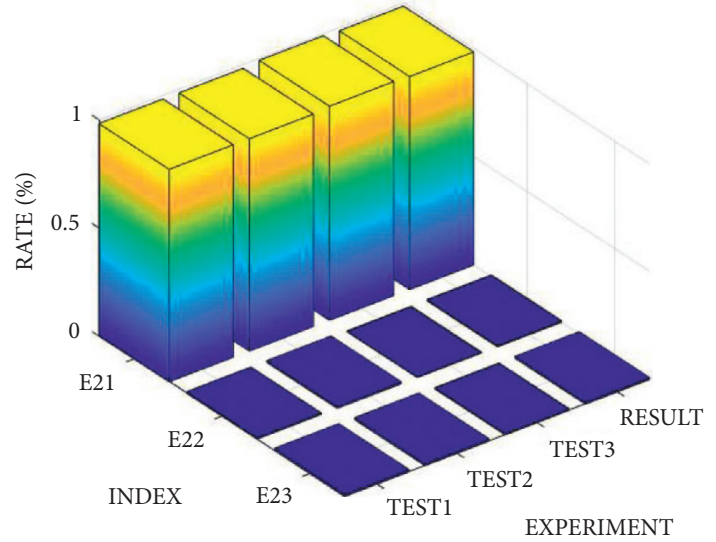

Figure 12: Proportion of each index of closed curve section.

Table 5: Proportion of each index of vehicle entry section.

\begin{tabular}{lccc}
\hline & $(\mathrm{E} 31)$ & $(\mathrm{E} 32)$ & $(\mathrm{E} 33)$ \\
\hline Test1 & 0.121438 & 0.766066 & 0.121438 \\
Test2 & 0.208209 & 0.679298 & 0.112493 \\
Test3 & 0.221088 & 0.678273 & 0.100639 \\
Result & 0.183578 & 0.707879 & 0.111523 \\
RMSE & 0.044253 & 0.041146 & 0.008518 \\
\hline
\end{tabular}

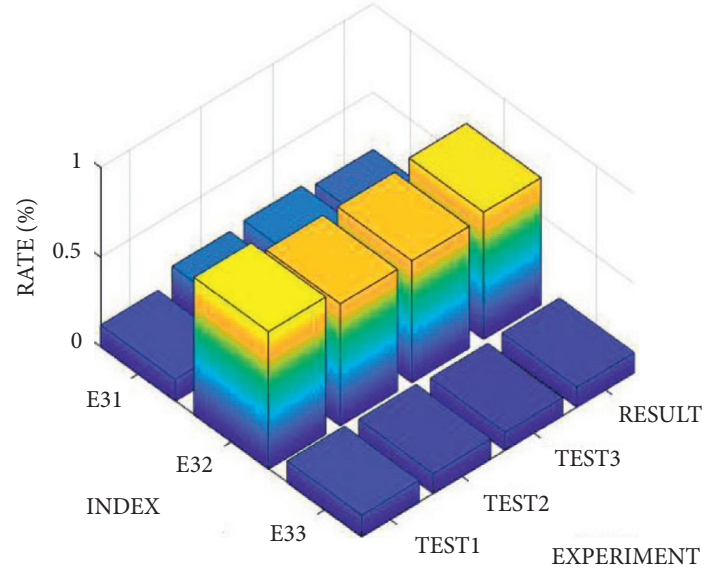

FIgURE 13: Proportion of each index of vehicle entry section.

TABLE 6: Evaluation results of vehicles leaving the road section.

\begin{tabular}{lccc}
\hline & $(\mathrm{E} 41)$ & $(\mathrm{E} 42)$ & $(\mathrm{E} 43)$ \\
\hline Test1 & 0.133483 & 0.743416 & 0.123100 \\
Test2 & 0.209290 & 0.683447 & 0.107264 \\
Test3 & 0.225745 & 0.666353 & 0.107902 \\
Result & 0.189506 & 0.697738 & 0.112755 \\
RMSE & 0.040179 & 0.033044 & 0.007319 \\
\hline
\end{tabular}

7.2. Real-Time Conflict Risk Assessment. Real-time assessments of vehicle conflict risk points on urban expressways may provide effective and intuitive urban expressway traffic safety risk points for traffic management departments in real time, facilitate police force distribution and road traffic

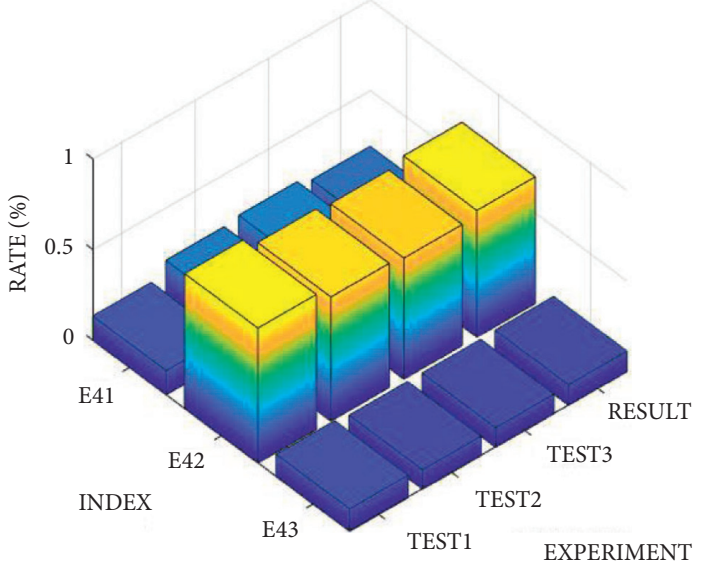

FIgURE 14: Proportion of various indicators of vehicles leaving the road section.

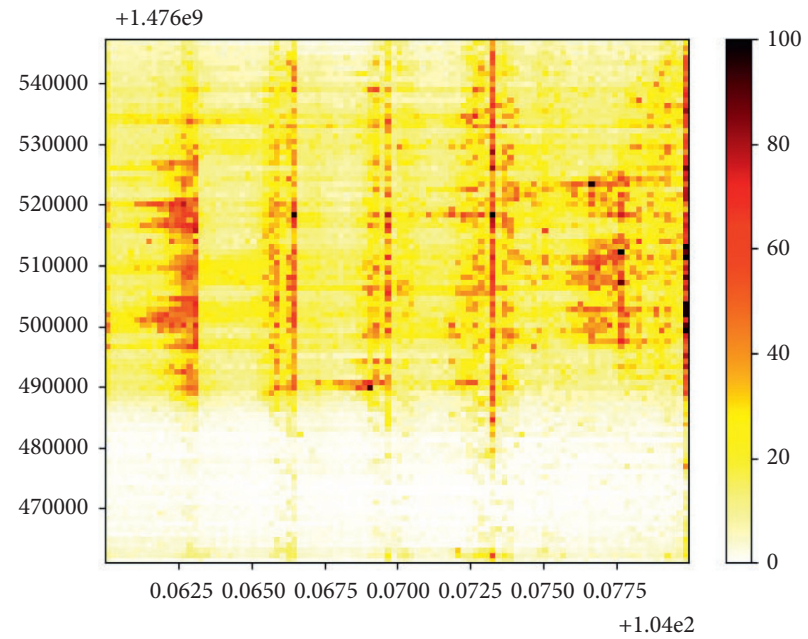

FIGURE 15: Daily variation of vehicle conflict risk in partially closed straight sections.

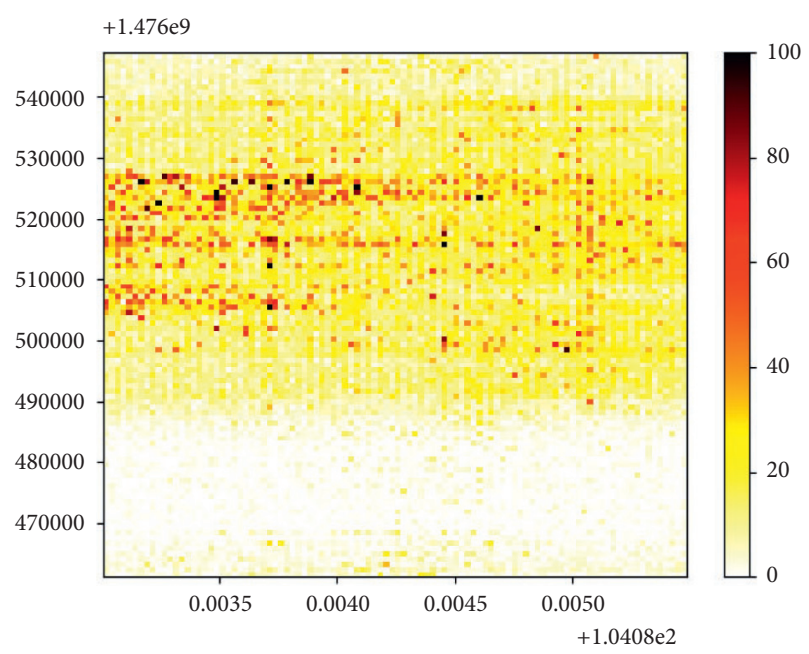

FIGURE 16: Daily variation of vehicle conflict risk in closed curve sections. 


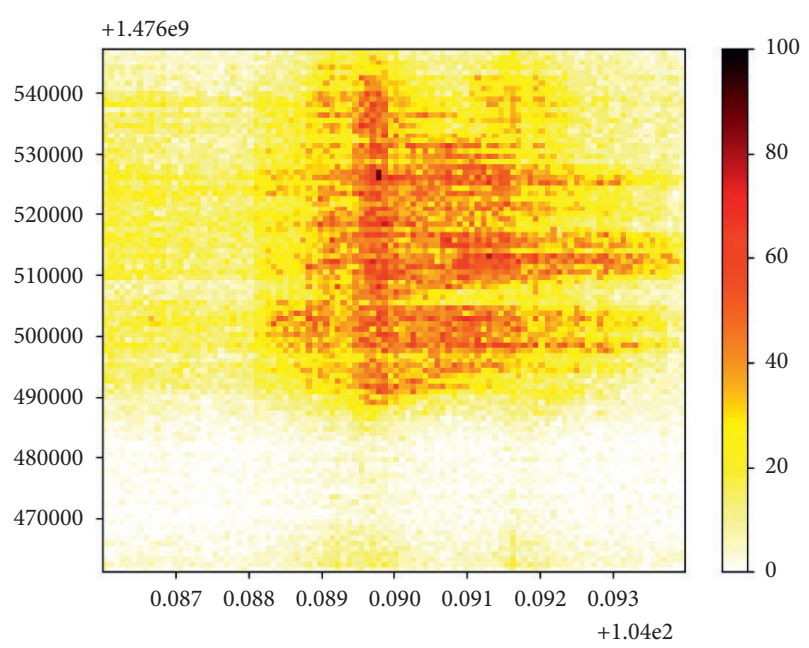

FIGURE 17: Daily variation of vehicle conflict risk in vehicle inflow section.

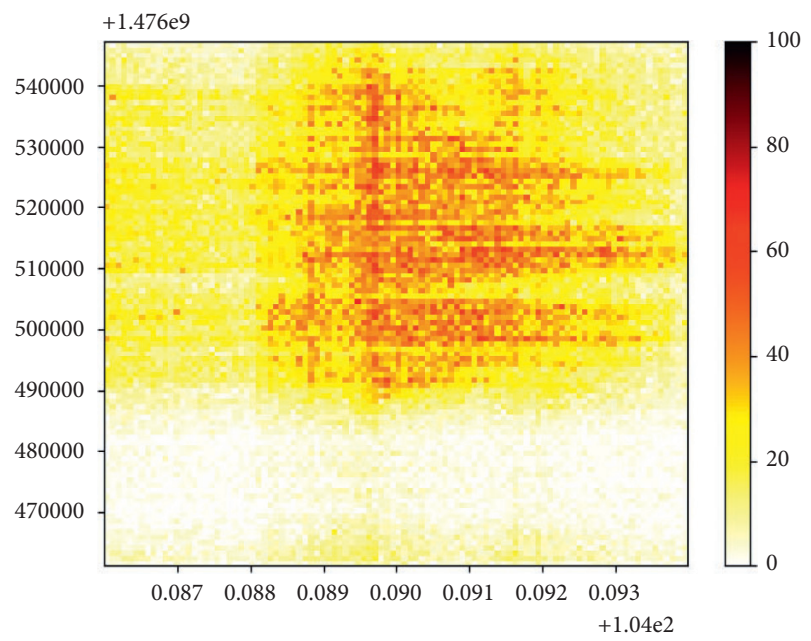

FIGURE 18: Daily variation of vehicle conflict risk in vehicle exit section.

control, and ultimately improve the traffic safety service level of urban expressways.

In the closed straight section at the north end of the Second Ring Expressway in Chengdu city, the coordinates and risk levels of road traffic safety risks were mostly concentrated in the locations with large vehicle speed and large changes in acceleration and deceleration. Vehicle conflict risk points and risk levels are shown in Figure 19.

In the closed curve section in the northeast of the Second Ring Expressway in Chengdu city, the coordinates and risk levels of road traffic safety risks were mostly concentrated in positions where the curve curvature was large and vehicles were about to enter the curve. Vehicle conflict risk points and risk levels are shown in Figure 20.

In the northeast part of the second Ring Expressway in Chengdu city, the coordinates and risk levels of traffic safety risks were mostly concentrated near the center of the intersection between the vehicle entrance and the trunk road.

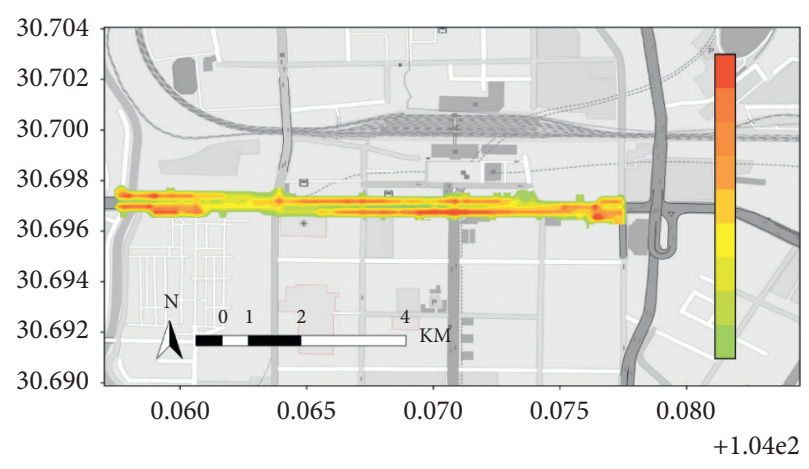

FIGURE 19: Safety assessment of closed straight section.

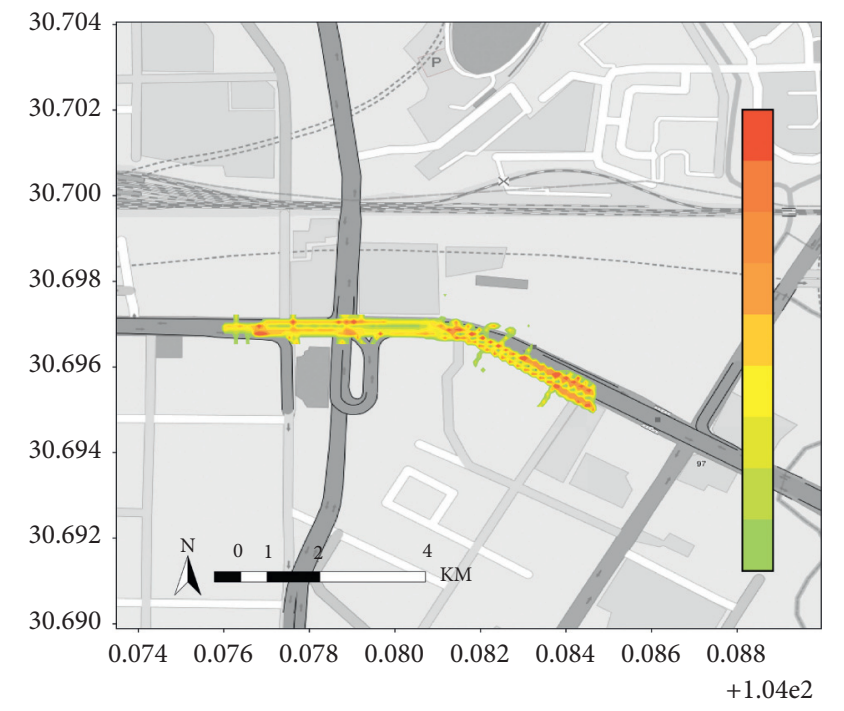

Figure 20: Safety assessment of closed curve section.

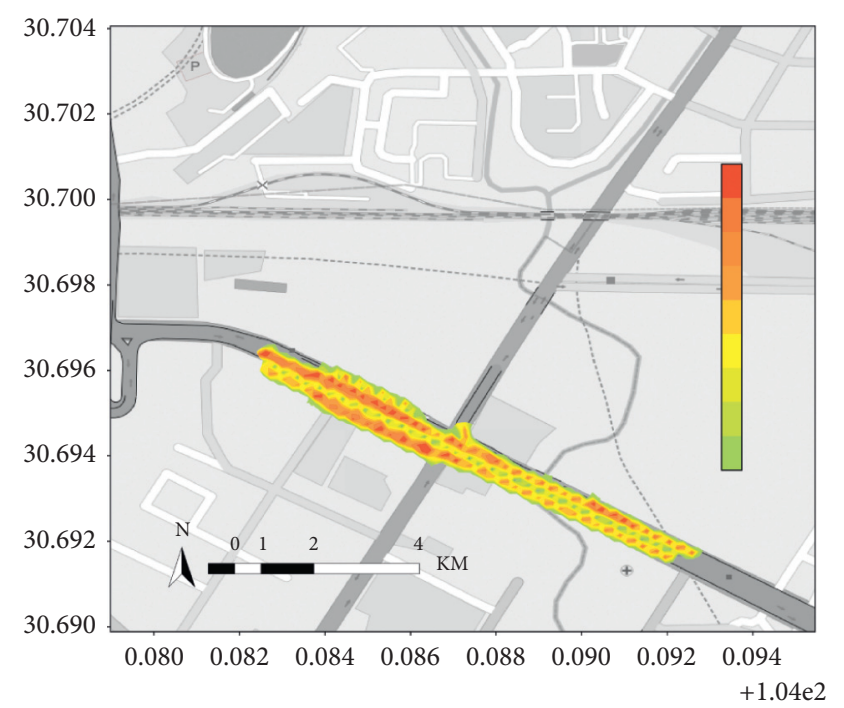

FIGURE 21: Safety assessment of vehicle entry section and vehicle exit section. 


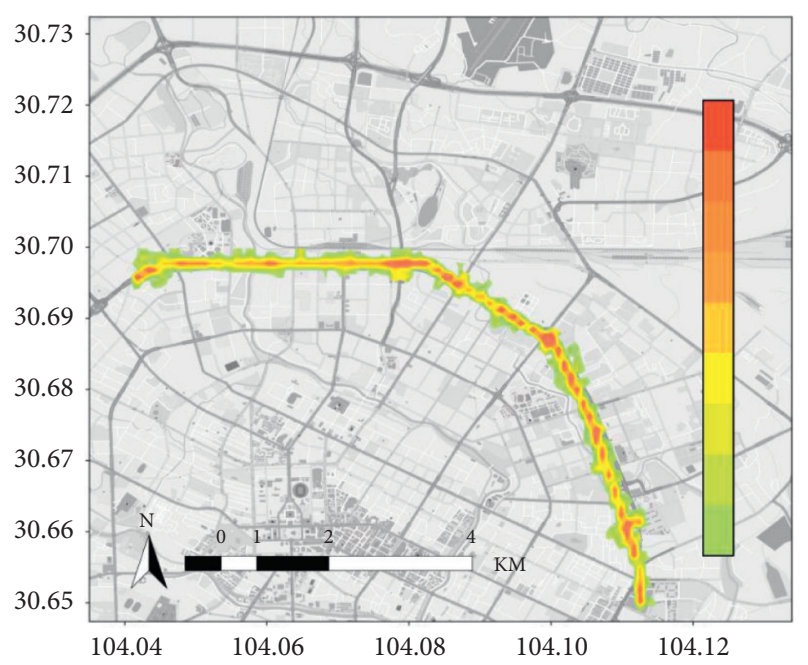

Figure 22: Safety assessment of second ring expressway in Chengdu city.

Vehicle conflict risk points and risk levels are shown in Figure 21.

In Chengdu city on expressway in the east and north road, all of the coordinates of road traffic safety risk and risk grade primarily concentrated in the straight sections of the vehicle speed or speed rate larger flat road center position, the bent section curvature maximum limit position, and vehicle location near out of conflict. Vehicle conflict risk points and risk levels are shown in Figure 22.

\section{Conclusions}

(1) This article proposed an extraction and analysis method of smartphone GPS data to obtain the realtime vehicle movement behavior of an urban expressway. According to the vehicle behavior and traffic characteristics of various sections of an urban expressway, the evaluation index of vehicle conflict behavior and the risk entropy evaluation model of vehicle conflict on urban expressway were constructed.

(2) Based on the GPS positioning data of smartphones of ride-hailing drivers in Chengdu city, this study evaluated the risk of vehicle conflict on the second ring road in Chengdu city in real time and determined the risk points of vehicle conflict in the north and east of the second ring expressway in Chengdu city using the constructed evaluation index of vehicle conflict behavior and the risk entropy evaluation model of vehicle conflict.

(3) The road traffic safety risk prediction system constructed only considered relevant situations in fast urban sections, and it is not applicable to general urban roads or expressways. Future work should include the influence of vehicle behavior and road traffic safety on different types of roads and sections as objectives. The conclusions section should clearly explain the main findings and implications of the work, highlighting its importance and relevance.

\section{Data Availability}

The data used to support the finding of this study are available from the corresponding author upon request.

\section{Conflicts of Interest}

The author declares no conflicts of interest.

\section{Acknowledgments}

The author thanks Prof. Fuquan Pan for his help in this research. This work was supported in part by Opening Foundation of Zhejiang Intelligent Transportation Engineering Technology Research Center, in part by the Shandong Provincial Key Research and Development Project of China under Grant 2018GGX105009, in part by the Humanities and Social Sciences Research Planning Foundation of Chinese Ministry of Education under Grant 18YJAZH067, in part by the Natural Science Foundation of Shandong Province of China under Grant ZR2020MG021, and in part by the National Natural Science Foundation of China under Grant 51505244.

\section{References}

[1] H. Zhang, S. Li, C. Wu, Q. Zhang, and Y. Wang, "Predicting crash frequency for urban expressway considering collision types using real-time traffic data," Journal of Advanced Transportation, vol. 2020, Article ID 8523818, 8 pages, 2020.

[2] R. Yu, Y. Wang, M. Quddus, J. Li, X. Wang, and Y. Tian, "Investigating vehicle roadway usage patterns on the Shanghai urban expressway system and their impacts on traffic safety," International journal of sustainable transportation, vol. 15, no. 3, pp. 217-228, 2021.

[3] W. Sun, "Major causes \& improvement suggestions to congestion in Shanghai Urban expressway," China Municipal Engineering, vol. 3, pp. 9-11, 2014.

[4] Transportation Bureau, Ministry of Public Security, The Road Traffic Accidents Statistics Report in China, Transportation Bureau, Ministry of Public Security, Beijing, China, 2017.

[5] M. Liu and Y. Chen, "Predicting real-time crash risk for urban expressways in China," Mathematical Problems in Engineering, vol. 2017, Article ID 6263726, 10 pages, 2017.

[6] J. Sun, T. Li, F. Li, and F. Chen, "Analysis of safety factors for urban expressways considering the effect of congestion in Shanghai, China," Accident Analysis \& Prevention, vol. 95, pp. 503-511, 2016.

[7] M. Strickland, G. Fainekos, and H. B. Amor, "Deep predictive models for collision risk assessment in autonomous driving," in Proceedings of the 2018 IEEE International Conference on Robotics and Automation (ICRA), pp. 4685-4692, IEEE, Brisbane, Australia, May 2018.

[8] F. Basso, L. J. Basso, F. Bravo, and R. Pezoa, "Real-time crash prediction in an urban expressway using disaggregated data," Transportation Research Part C: Emerging Technologies, vol. 86, pp. 202-219, 2018.

[9] M. Hossain and Y. Muromachi, "A real-time crash prediction model for the ramp vicinities of urban expressways," IATSS Research, vol. 37, no. 1, pp. 68-79, 2013. 
[10] H. M. Hassan and M. A. Abdel-Aty, "Predicting reduced visibility related crashes on freeways using real-time traffic flow data," Journal of Safety Research, vol. 45, pp. 29-36, 2013.

[11] J. Hong, R. Tamakloe, and D. Park, "A comprehensive analysis of multi-vehicle crashes on expressways: a double hurdle approach," Sustainability, vol. 11, no. 10, p. 2782, 2019.

[12] G. Fountas, M. T. Sarwar, P. C. Anastasopoulos, A. Blatt, and K. Majka, "Analysis of stationary and dynamic factors affecting highway accident occurrence: a dynamic correlated grouped random parameters binary logit approach," Accident Analysis \& Prevention, vol. 113, pp. 330-340, 2018.

[13] A. Kassu and M. Hasan, "Factors associated with traffic crashes on urban freeways," Transport Engineer, vol. 2, Article ID 100014, 2020.

[14] L. Wang, M. Abdel-Aty, J. Lee, and Q. Shi, "Analysis of realtime crash risk for expressway ramps using traffic, geometric, trip generation, and socio-demographic predictors," Accident Analysis \& Prevention, vol. 122, pp. 378-384, 2019.

[15] C. Wu, L. Peng, Z. Huang, M. Zhong, and D. Chu, "A method of vehicle motion prediction and collision risk assessment with a simulated vehicular cyber physical system," Transportation Research Part C: Emerging Technologies, vol. 47, pp. 179-191, 2014.

[16] F. G. Habtemichael and L. de Picado Santos, "Crash risk evaluation of aggressive driving on motorways: microscopic traffic simulation approach," Transportation Research Part F: Traffic Psychology and Behaviour, vol. 23, pp. 101-112, 2014.

[17] C. Katrakazas, M. Quddus, and W.-H. Chen, "A new integrated collision risk assessment methodology for autonomous vehicles," Accident Analysis \& Prevention, vol. 127, pp. 61-79, 2019.

[18] M. Hosseinpour, S. Sahebi, Z. H. Zamzuri, A. S. Yahaya, and N. Ismail, "Predicting crash frequency for multi-vehicle collision types using multivariate Poisson-lognormal spatial model: a comparative analysis," Accident Analysis \& Prevention, vol. 118, pp. 277-288, 2018.

[19] F. Chen, S. Chen, and X. Ma, "Analysis of hourly crash likelihood using unbalanced panel data mixed logit model and real-time driving environmental big data," Journal of Safety Research, vol. 65, pp. 153-159, 2018.

[20] X. Chen, H. Chen, Y. Yang et al., "Traffic flow prediction by an ensemble framework with data denoising and deep learning model," Physica A: Statistical Mechanics and Its Applications, vol. 565, Article ID 125574, 2021.

[21] J. Stipancic, L. Miranda-Moreno, and N. Saunier, "Vehicle manoeuvers as surrogate safety measures: extracting data from the gps-enabled smartphones of regular drivers," Accident Analysis \& Prevention, vol. 115, pp. 160-169, 2018.

[22] X. Cai, C. Lei, B. Peng, X. Tang, and Z. Gao, "Road traffic safety risk estimation method based on vehicle onboard diagnostic data," Journal of Advanced Transportation, vol. 2020, Article ID 3024101, 13 pages, 2020.

[23] D. Babić and T. Brijs, "Low-cost road marking measures for increasing safety in horizontal curves: a driving simulator study," Accident Analysis \& Prevention, vol. 153, Article ID 106013, 2021.

[24] K. G. Qian, "Research on mechanical conditions of vehicle rollover at corners," Advanced Materials Research, vol. 1046, pp. 187-190, 2014.

[25] R. S. Tomar and S. Verma, "Safety of lane change maneuver through A priori prediction of trajectory using neural networks," Network Protocols and Algorithms, vol. 4, no. 1, pp. 4-21, 2012.
[26] Y. Zhou, M. Deng, and N. C. Lu, "Analysis of driving behavior and safety in urban expressway," Traffic Information and Safety, vol. 1, pp. 7-12, 2019.

[27] C. E. Shannon and W. Weaver, The Mathematical Theory of Communication, The University of Illinois Press, Urbana, IL, USA, 1947.

[28] W. Huang, B. Shuai, Y. Sun, Y. Wang, and E. Antwi, "Using entropy-TOPSIS method to evaluate urban rail transit system operation performance: the China case," Transportation Research Part A: Policy and Practice, vol. 111, pp. 292-303, 2018. 